\title{
Terapia ocupacional na abordagem de pessoas em tratamento por anorexia
}

nervosa

\author{
Aline Midori Adati Kubota* Ana Cláudia Reis de Magalhães** \\ Vagner Dos Santos ${ }^{* * *}$ Andrea Donatti Gallassi ${ }^{* * * *}$
}

Resumo: A Anorexia Nervosa (AN) foi o primeiro distúrbio alimentar a ser estudado no século XIX e possui etiologia multifatorial. Os indivíduos com AN apresentam traços de perfeccionismo, baixa autoestima, ansiedade, introversão e baixo peso corporal. 0 objetivo deste estudo foi o de realizar um estudo teórico sobre a anorexia nervosa e suas implicações no cotidiano do indivíduo que sofre da doença discutindo as possibilidades de intervenção da Terapia Ocupacional. A estratégia de pesquisa utilizada foi a de revisão bibliográfica que foi realizada nas bases de dados eletrônicas: BIREME, LILACS, MEDLINE, PUBMED e SCIELO, utilizando-se os descritores Anorexia Nervosa e Terapia Ocupacionalcombinados, e os mesmo em inglês. A amostra foi de 32 artigos, sendo que para elaboração deste trabalho foram utilizados 11. Destes, apenas sete estudos listaram descritivamente as intervenções da Terapia Ocupacional na assistência aos pacientes com AN. Não foi encontrado nenhum estudo sobre eficácia da intervenção do terapeuta ocupacional no tratamento de indivíduos com AN e nem publicações recentes e específicas sobre o tema, nem mesmo na literatura estrangeira, 0 que sugere que mais pesquisas científicas sobre o tema sejam realizadas e publicadas

Descritores: Anorexia Nervosa, Terapia Ocupacional, Saúde Mental

\section{Occupational therapy approach in people in treatment for anorexia nervosa}

Abstract: Anorexia Nervosa (AN) was the first eating disorder to be studied in the nineteenth century. It has multifactorial etiology and individuals suffering from AN exhibit trait of perfectionism, low selfesteem, anxiety, introversion and low body weight. The objective of this study was to carry out a theoretical study on the anorexia nervosa and its implications in the individual daily life anddiscuss the possibilities of Occupational Therapyintervention.A literature review was performed in the following electronic databases: BIREME, LILACS, MEDLINE, PUBMED and SCIELO, using the descriptors 'Anorexia Nervosa' and 'Occupational Therapy', in both English and Portuguese. 32 articleswere found, being 11 articles used in this paper.Among them, only seven studies focused on the Occupational Therapy interventions. There has been no study on effectiveness of occupational therapistinterventionsin the treatment of individuals with AN. Finally, it was not found recent and specific publications on the subject, not even in foreign literature. This suggests that more scientific research on the topic are need.

Descriptors: Anorexia Nervosa, OccupationalTherapy,

*Graduanda em Terapia Ocupacional na Universidade de Brasília (UNB), Brasília, DF, Brasil.

**Mestre em Psicologia Clínica e Cultura pela Universidade de Brasília (UNB), Brasília, DF, Brasil.

***Mestre em Health and Society pela Linköping University (LiU),Linköping, Suécia.

****Doutora em Psiquiatria pela Universidade de São Paulo (USP), São Paulo, SP, Brasil. 


\section{Introdução}

Os transtornos alimentares são patologias graves e complexas que constituem quadros psiquiátricos caracterizados pelo comportamento alimentar alterado. Sua etiologia é multifatorial, envolvendo fatores predisponentes, precipitantes e mantenedores do transtorno que irão interagir entre si para produzir e lou perpetuar a patologia,sendo a anorexia nervosa (AN) um dos tipos principais de transtorno alimentar. ${ }^{1-3}$

A AN foi o primeiro transtorno alimentar a ser estudado no século XIX, e em 1970 pioneiro na sua classificação e a ter estabelecido critérios operacionais.4Um dos primeiros relatos descritos foium caso sugestivo de anorexia nervosa em uma serva que viveu no ano de 1985 e que veio a óbito por desnutrição. ${ }^{5}$

Outras obras, apresentam a etiologia da AN relacionada com o quadro de histeria. No entendimento da psicanálise, a ausência de apetite alimentar simboliza para a anoréxica a falta do apetite sexual e a sua ocupação exclusiva com as questões relacionadas com a alimentação está direcionada para as questões relacionadas com a sexualidade. ${ }^{6}$

Segundo o Manual Diagnóstico e Estatístico de Transtornos Mentais (DSM-IV-TR), $4^{\text {a }}$ edição, a AN é caracterizada por I) recusa em manter o peso dentro ou acima do mínimo normal adequado à idade e à altura; II) medo intenso do ganho de peso ou de se tornar gordo, mesmo com peso inferior;/II) perturbação no modo de vivenciar o peso, tamanho ou formas corporais; IV) na mulher, a ausência de pelo menos três ciclos menstruais consecutivos, quando é esperado ocorrer o contrário (amenorreia primária ou secundária). ${ }^{7}$

ODSM-IV-TRclassifica a AN em dois tipos: Anorexia nervosa do tipo restritivo, em que não existe episódio de comer compulsivamente ou prática purgativa (vômito auto induzido, uso de laxantes, diuréticos e enemas) e a Anorexia nervosa do tipo purgativo em que há episódio de comer compulsivamente e lou purgação.

Já a Classificação Internacionalde Doenças (CID-10) define a AN quando: I) há perda de peso ou, em crianças, falta de ganho de peso; II) o peso corporal é mantido em pelo menos $15 \%$ abaixo do esperado, ou seja, um índice de massa corporal (IMC) igual ou abaixo de 17,$5 ;$;II) a perda de peso é auto induzida; existe uma distorção na imagem corporal na forma de uma psicopatologia específica de um pavor de engordar; e IV) presença de um transtorno endócrino generalizado envolvendo o eixo-hipotalâmico-hipofisário-gonadal, que é manifestado em mulheres como amenorreia e em homens como uma perda de interesse e potências sexuais. ${ }^{8}$

É conhecido que traços como obssessividade,perfeccionismo, passividade e introversão são comuns em pacientes com AN e permanecem estáveis mesmo após a recuperação do peso. ${ }^{9,10}$ Entre os fatores predisponentes individuais para a AN estão: Traços de personalidade (baixa auto-estima, traços obsessivos e perfeccionistas), história de transtornos psiquiátricos (depressão e transtornos da ansiedade), tendência à obesidade, alterações da neurotransmissão (vias noradrenérgicas e vias serotoninérgicas) e eventos adversos como abuso sexual.11A agregação familiar, hereditariedade e padrões de interação familiar (rigidez, intrusividade e evitação de conflitos) como fatores predisponentes familiares; e o ideal cultural de magreza como fator sociocultural predisponente.Além das características

Saúde (Santa Maria), v.39, n.2, p.23-34, 2013 Terapia ocupacional na abordagem de pessoas em tratamento por anorexia nervosa apresentadas pelo quadro da anorexia nervosa, esta pode provocar um grave estado de inanição e desencadear distúrbios endocrinológicos, gastrointestinais,cardiovasculares, hematológicos e metabólicos que contribuem para a manutenção e cronificação do quadro, além de favorecer o desenvolvimento de patologias secundárias. Os sintomas associados 
podem resultar em caquexia (fraqueza geral do corpo e má disposição corporal decorrente da desnutrição). ${ }^{11,12}$

A AN acomete o sexo feminino em cerca de $95 \%$ das ocorrências, principalmente entre jovens de 14 e 17 anos, sendo que pode ocorrer tanto precocemente, quanto tardiamente. Quando a AN está associada com o diagnóstico e tratamento tardio sua taxa de mortalidade é de aproximadamente $5 \%$.Porém essa patologia também acomete homens e crianças de ambos os sexos. ${ }^{4,13}$

Nesse sentido, devido à anorexia nervosa ser uma das patologias em que ocorre 0 comprometimento do convívio social, vínculos familiarese desorganização do cotidiano,é importante a participação de diferentes profissionais da saúde atuando no processoseu tratamento.No entanto, este éum campo de atuação recente com poucos e com estudos publicados sobre a atuação de diferentes profissões, como a Terapia Ocupacional. Sendo assim, nosso objetivo foi realizar uma revisão teórica sobre a anorexia nervosa e suas implicações no cotidiano do indivíduo anoréxico erelacionar e discutir as possibilidades de intervenção da Terapia Ocupacional, considerando suas intervenções na área da saúde, educação e social, que visam a autonomia e produção de cuidado do sujeito em suas ações

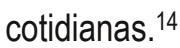

\section{Metodologia}

Foi realizada uma pesquisa bibliográfica, cujo objetivo foi o levantamento demanuscritos já publicada, em forma de artigos científicos. A finalidade desta metodologia é fazer com que o pesquisador entre em contato direto com o material escrito sobre um determinado assunto, auxiliando o cientista na análise de suas pesquisas e na manipulação de suas informações. ${ }^{15}$ Assim, foi realizadas buscas nas bases de dados eletrônicas: Biblioteca Virtual em Saúde (BVS), Literatura Latino-Americana e do Caribe em Ciências da Saúde (LILACS), National Library of Medicine (MEDLINE), National Library of Medicine Nationallnstituteof Health(PubMed) e ScientificEletronic Library Online (SciELO), restringindo a busca para artigo publicados entre 1985 e 2012. Este período foi utilizado para abranger estudos importantes para elaboração do trabalho e a pequena quantidade de estudos publicados sobre o tema. Os descritores foram utilizados de forma associada: anorexia nervosa e terapia ocupacional e os seus correspondentes em idioma inglês (anorexia nervosa andoccupationaltherapy). Realizou-se também uma leitura e revisão dos artigos citados pelos autores dos artigos selecionados.

Foram incluídos artigos em português e inglês escritos por terapeutas ocupacionais ou por outros profissionais da área da saúde que citassem o terapeuta ocupacional como membro da equipe multidisciplinar,e foram excluídos todos aqueles que não tratassem do tema ou fossem repetidos.

Os dados levantados foram organizados em duas planilhas eletrônicas. A primeira planilha auxiliou no tratamento dos aspectos específicos do tema, tais como etiologia da AN, critérios diagnósticos, tratamento básico, importância e intervenções de outros profissionais da saúde, trabalho em equipe, avaliação da TO, reavaliação da equipe multidisciplinar

Rev. Saúde (Santa Maria), Santa Maria, v.39, n.2 p.25-34, Jul./Dez.2013 estabelecimento de uma relação terapêutica, estágio de negação e aceitação da AN, intervenções da TO, papel da TO e principais áreas afetadas. 
A segunda planilha consistiu na abordagem dos aspectos metodológicos dos estudos encontrados como: ano de publicação, país, área do autor principal, tipo de estudo, instrumento de pesquisa e resultados importantes; em que possibilitou o mapeamento dos estudos realizados sobre o tema.

Primeiramente, os dados coletados foram tratados de forma qualitativa com o auxílio da primeira planilha e posteriormente de forma quantitativa baseada na segunda planilha.

Realizou-se uma leitura seletiva, na qual os artigos eram previamente selecionados durante a pesquisa bibliográfica. Executou-se a verificação do título, leitura do resumo ou do estudo na íntegra para seleção do material. As informações utilizadas na análise quantitativa foram encontradas pela busca das informações contidas nas notas de rodapé ou após a leitura do texto completo do artigo. Posteriormente, efetuou-se uma leitura crítica para análise do conteúdo dos dados encontrados e em seguida, uma leitura interpretativa desses dados.

Os dados qualitativos foram apresentados a partir da análise do conteúdo utilizando-se a abordagem narrativa e os dados quantitativos pela frequência dos resultados obtidos com enfoque matemático simples, utilizando-se como instrumentos gráficos e tabelas.

\section{Resultados e discussão}

Foram encontrados 32 artigos, sendo que para a elaboração deste trabalho foram utilizados apenas 11 artigos que contemplavam os critérios de inclusão.As publicações possuem maior frequência no ano de 2006. Sendo que seis artigos possuem como país de origem o Brasil, quatro artigos do Reino Unido/Inglaterra e Argentina. (Tabela 1)

Tabela 1 - Amostra de artigos científicos do estudo

\begin{tabular}{|c|c|c|c|}
\hline Autor(es) & Título & Ano & Origem (Idioma) \\
\hline GILES, G.M. & Anorexia Nervosa and Bulimia: An Activity-Oriented Approch & 1995 & $\begin{array}{c}\text { Reino Unido/ } \\
\text { Inglaterra (Inglês) }\end{array}$ \\
\hline MARTIN, J.E. & Occupational Therapy in Anorexia Nervosa & 1985 & $\begin{array}{c}\text { Reino Unido/ } \\
\text { Inglaterra (Inglês) }\end{array}$ \\
\hline $\begin{array}{l}\text { GILES, G.M.; } \\
\text { ALLEN, M. E. }\end{array}$ & $\begin{array}{l}\text { Occupational Therapy in the Rehabilitation of the Patient with } \\
\text { Anorexia Nervosa }\end{array}$ & 1986 & $\begin{array}{c}\text { Reino Unido/ } \\
\text { Inglaterra (Inglês) }\end{array}$ \\
\hline $\begin{array}{l}\text { ESTURARO, } \\
\text { A. T. A. et al. }\end{array}$ & $\begin{array}{l}\text { Uma proposta de intervenção do terapeuta ocupacional no } \\
\text { tratamento da anorexia nervosa }\end{array}$ & 1998 & Brasil (Português) \\
\hline $\begin{array}{l}\text { HENDERSON, } \\
\text { S. }\end{array}$ & $\begin{array}{l}\text { Frames of reference utilized in the rehabilitation of individuals with } \\
\qquad \text { eating disorders }\end{array}$ & 1999 & $\begin{array}{c}\text { Reino Unido/ } \\
\text { Inglaterra (Inglês) }\end{array}$ \\
\hline $\begin{array}{l}\text { MATUSEVICH, } \\
\text { D. et al. }\end{array}$ & $\begin{array}{l}\text { Hospitalization of patients with anorexia nervosa: a therapeutic } \\
\text { proposal }\end{array}$ & 2002 & Argentina (Inglês) \\
\hline $\begin{array}{l}\text { SOUZA, J. C. } \\
\text { et al. }\end{array}$ & Transtornos Alimentares em Discussão Multidisciplinar & 2002 & Brasil (Português) \\
\hline MORAIS, L. V. & $\begin{array}{c}\text { A assistência do terapeuta ocupacional para pessoas com } \\
\text { anorexia nervosa: relato de experiência }\end{array}$ & 2006 & Brasil (Português) \\
\hline
\end{tabular}

Saúde (Santa Maria), v.39, n.2, p.23-34, 2013. Terapia ocupacional na abordagem de pessoas em tratamento por anorexia nervosa anorexia nervosa: relato de experiência 
CABRERA, C.

C.

SILVA, L. M.; SANTOS, M. A.

QUILESCESTARI, L.M.

et al.

Como um dos descritores para a busca dos artigos foi "terapia ocupacional", a maioria dos que foram selecionados tem o terapeuta ocupacional como um dos autores,sendo na sua grande parte revisões de literatura. (Gráfico 1)

Gráfico 1 - Tipo de estudo e Formação do autor principal

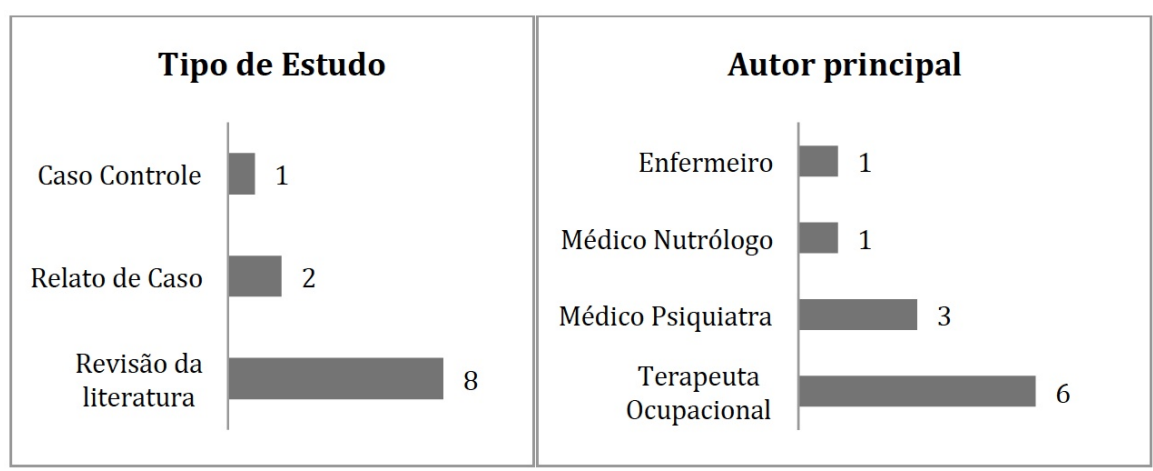

Observam-se nos dados coletados que apenas dois artigos ${ }^{16,17}$ apresentaram 0 critério diagnóstico de AN baseado no DSM-IV-TR e nenhum apresentou a definição segundo a CID10. Os estudos apresentaram definições baseadas em estudos de outros autores que estudam sobre o tema.

Embora não usem os mesmo critérios, a etiologia apresentada pelos estudos foi consensual, e definida como multifatorial compreendendo fatores genéticos, biológicos, socioculturais, familiares, personalidade e vulnerabilidade psicológica e fatores individuais como alguma experiência adversa. ${ }^{16-26}$

Dos 11 artigos, quatro artigos abordavam como tratamento básico para AN a recuperação de peso normal, o tratamento nos serviços de alta complexidade, atuação da equipe multidisciplinar e o tratamentodividido em duas partes: a primeira, uma intervenção do profissional médico com a finalidade de estabilização da condição física do paciente anoréxico, e a segunda parte que consiste no apoio para que o paciente estabilize seu peso e que sejam trabalhados os problemas apresentados através da psicoterapia. Sendo que os autores apontam que a intervenção de Terapeuta Ocupacionais é mais efetiva nessa segunda parte do tratamento. ${ }^{16,19,23}$

Muitos autores citaram a importância da equipe multidisciplinar composta por enfermeiro, psicólogo, médico psiquiatra, nutricionista, assistente social e terapeuta ocupacional. ${ }^{16-17,19-26}$ Sendo que expõem algumas intervenções desses profissionais tais como: terapia familiar abordagem psicanalítica, abordagem cognitiva comportamental, grupo de orientação médiconutricional, grupo de apoio psicológico para os cuidadores dos anoréxicos, prescrição e administração de medicamentos, cuidados durante a internação como aferição dos sinais
Rev. Saúde (Santa Maria), Santa Maria, v.39, n.2, p.25-34, Jul./Dez.2013 Kubota, A. M.A; et. al. 
vitais, administração de dieta, observação dos aspectos psicossociais, dentre outras intervenções.

Um dos artigos selecionados não descreveu a importância do trabalho em equipe por se tratar de um artigo mais específico sobre a Terapia ocupacional no tratamento da $\mathrm{AN}^{18}$. Os demais autores dos estudos abordaram a importância da reunião de equipe e boa comunicação entre os profissionais como fatores positivos no tratamento da AN.

Cinco artigos abordam a importância do estabelecimento de uma relação terapêutica/vínculo, em que esta deve consistir em uma boa relação do terapeuta ocupacional com o paciente, estabelecimento de um contrato de tratamento com o paciente e essa relação do mesmo com o terapeuta deve ser de confiança. ${ }^{16-19,25}$

$O$ estágio de negação e aceitação da AN é descrito por alguns autores como um fator observado no discurso do paciente, apresentados pela dificuldade de compreender e responder as mensagens que recebem do corpo, em que a dificuldade de aceitação da AN reflete na não adesão ao tratamento e o anoréxico é levado ao serviço de saúde contrariado e pouco motivado para mudança de seu comportamento. ${ }^{16-17,19,22}$ Alguns anoréxicos entram no estágio de aceitação a partir do momento que entram em contato com o serviço de saúde.

Também é considerado em algumas publicações, que o primeiro passo para intervenção da Terapia Ocupacional a avaliação do paciente anoréxico e que a mesma deve ser contínua. Citam avaliações tais como a lista de identificação dos papéis ocupacionais do indivíduo e entrevista semi-estruturada. ${ }^{16,19,26}$

A lista de identificação dos papéis ocupacionais foi criada pelo terapeuta ocupacional, Francis Oakley, com o objetivo de coletar informações sobre os papéis ocupacionais do paciente. Possui como públicos-alvo adolescentes, adultos e idosos. Divide-se em duas partes: a parte I avalia os principais papéis ocupacionais que compõem a vida diária do paciente e a parte II menciona o grau de importância que o mesmo atribui a cada papel. A lista apresenta dez papéis ocupacionais (estudante, trabalhador, voluntário, cuidador, serviço doméstico, amigo, membro de família, religioso, passatempo/amador e participante em organizações) pré-definidos, acrescido da opção outro em que se pode listar algum outro papel ocupacional que não tenha sido listado, e a lista é um instrumento auto aplicável. ${ }^{27}$

A avaliação é indicada como o primeiro passo para o tratamento da AN e a primeira avaliação deve ser feita em formato de entrevista. Esta deve ser composta de fatores biológicos, sociais e psicológicos; em que devem ser observados os excessos comportamentais do indivíduo, déficits comportamentais e comportamentos ativos. As principais áreas que devem ser abordadas na entrevista são as atividades de vida diária, competências sociais, déficits cognitivos, trabalho e lazer. ${ }^{19} O$ terapeuta ocupacional além de entrevistar o paciente para coleta de informações também pode entrevistar familiares e/ou amigos se considerar necessário.Importante considerar que, conforme alguns autores, nenhuma atividade em si é terapêutica para o paciente com AN. ${ }^{16,19}$ É necessário o empenho e esforço do terapeuta ocupacional e do paciente para elaborarem atividades que irão produzir mudanças no comportamento do paciente anoréxico, sendo que há uma variedade de estratégias de intervenção. Nesse sentido, segundo esses autores o Terapeuta Ocupacional conta com uma diversidade de intervenções no processo de cuidado de pessoas com anorexia nervosa (Tabela 2.). 
Tabela 2 - Intervenções da Terapia Ocupacional.

\begin{tabular}{|c|c|}
\hline Autor(es) & Intervenções \\
\hline GILES, G.M. & $\begin{array}{l}\text { Yoga, prática de cozinhar, arte terapêutica, treinamento de assertividade, grupo de } \\
\text { discussões, atividade de artesanato, educação/informação sobre a AN, grupo } \\
\text { cognitivo comportamental, ensinar estratégias comportamentais, psicodrama, compra } \\
\text { de roupas, uso de vídeos e acesso literatura sobre o tema. }\end{array}$ \\
\hline MARTIN, J.E. & $\begin{array}{l}\text { Autocuidado, cozinhar, grupo de atividades, atividades estruturadas e criativas, arte } \\
\text { projetada, sessões de beleza e maquiagem, continuidade do trabalho escolar, } \\
\text { atividades para melhorar a concentração, arte projetiva, corte e costura, relaxamento, } \\
\text { treinamento de habilidades sociais, planejamento de menu, compra de alimentos, } \\
\text { cozinhar, introdução de atividades físicas, fazer refeições fora do ambiente domiciliar. }\end{array}$ \\
\hline $\begin{array}{l}\text { GILES, G.M.; } \\
\text { ALLEN, M. E. }\end{array}$ & $\begin{array}{l}\text { Imagem corporal, yoga, técnicas de relaxamento, atividades orientadas, terapia } \\
\text { cognitiva, cozinhar, fazer compras, estratégias comportamentais, visita domiciliar, } \\
\text { grupo terapêutico, abordagem social, planejamento de exercícios, artesanato, arte } \\
\text { terapia. }\end{array}$ \\
\hline HENDERSON, S. & Abordagem psicanalítica e cognitivo- comportamental. \\
\hline SOUZA et al. & $\begin{array}{l}\text { Tratamento direcionado também para família e abordagem cognitivo } \\
\text { comportamental. }\end{array}$ \\
\hline MORAIS, L. V. & $\begin{array}{l}\text { Construir uma nova relação com seu fazer, por meio do planejar, organizar e realizar } \\
\text { junto com o terapeuta ocupacional suas ações cotidianas, fazer bijuterias, abordagem } \\
\text { de receitas culinárias e cozinhar na sessão com a presença da terapeuta ocupacional } \\
\text { e nutricionista. }\end{array}$ \\
\hline $\begin{array}{c}\text { QUILES- } \\
\text { CESTARI, L.M. et } \\
\text { al. }\end{array}$ & Trabalhar dificuldades relacionadas com o trabalho e relações interpessoais. \\
\hline
\end{tabular}

A arte terapia é um recurso utilizado como forma de autoexpressão de sentimentos. 0 terapeuta pode estimular essa atividade norteando temas como "Como eu me vejo", "O que eu sinto depois de uma refeição", "O que eu sinto no momento da refeição" e "Como me vejo no futuro" em que o paciente será estimulado a expressar esses sentimentos. ${ }^{16,18}$

0 treinamento de assertividade deve ser realizado em grupo em que o terapeuta ocupacional poderá ensinar 0 paciente a validar, apreciar e valorizar seus próprios sentimentos e aprender a controlar e compreender os seus impulsos que podem ser agressivos ao outro. Aspectos relacionados com as relações individuais e eliminação de atitudes consideradas prejudiciais também devem ser trabalhados nesse grupo, se utilizado exemplos concretos. ${ }^{16}$

As atividades de artesanato e confecção de bijuterias podem ser utilizadas para aumentar os sentimentos de eficácia e construção de confiança. Essas atividades auxiliam no exame cognitivo de pensamentos e estilos de pensamento, avaliação das reações do paciente ao fracasso e frustração (que são aspectos da vida cotidiana) e que a partir dessa demanda apresentada se pode elaborar e desenvolver técnicas de enfrentamento dessas reações. Assim como relatos de uma experiência de atendimento de um grupo de anoréxicos Rev. Saúde (Santa Maria), Santa Maria, v.39, n.2 p.25-34, Jul./Dez.2013. Kubota $A \cdot M$; et at no Serviço de Interconsulta em Saúde Mental do Hospital das Clínicas da Faculdade de 
Medicina de Ribeirão Preto-USP, utilizando-se a técnica de confecção de bijuterias proporcionou aos integrantes do grupo a vontade de querer sair de casa para comprar materiais e experimentar algo novo, estimulando a vida social desses pacientes. ${ }^{16,23}$

No grupo com abordagem cognitivo comportamental ajuda-se o paciente a identificar e tentar mudar as construções alteradas que faz da realidade e os comportamentos disfuncionais que emergem como resultado disso. Primeiramente deve-se trabalhar com os estilos de pensamento disfuncionais em que 0 anoréxico será estimulado a identificar esses pensamentos de modo a ampliar o significado dos acontecimentos em que estão inseridos e reavaliar de forma adequada e racional os acontecimentos do cotidiano. Em seguida trabalhase com a identificação e avaliação dos pensamentos automáticos referentes à imagem corporal, analisando suas vantagens e desvantagens. Também deve ser destacada a discussão sobre a identificação de cognições disfuncionais associadas ao comer, peso e forma corporal utilizando-se a técnica de desafio dos pensamentos expostos. Pode ser sugerido que os pacientes realizem um registro diário de pensamentos disfuncionais para que sejam trabalhados no grupo pelo terapeuta ocupacional. ${ }^{16,17}$

A visita domiciliar pode ser realizada para que sejam trabalhados problemas particulares de forma mais concreta, prática e de forma que o terapeuta ocupacional possa conhecer melhor a dinâmica da organização e relação familiar do paciente.

O psicodrama é ressaltado, quando utilizado por um terapeuta ocupacional treinado pode ser um recurso eficaz no tratamento da AN. ${ }^{16}$ Para realizar o psicodrama, devem-se conhecer os problemas vivenciados pelo paciente e este recurso deve ser utilizado no período final do tratamento. As técnicas utilizadas devem ser focadas nas necessidades do paciente, por exemplo, o uso de escultura da família do paciente pode demonstrar sua posição e papéis desempenhados na família.

A atividade no ambiente social, como por exemplo de comprar roupas junto com esses pacientes é um recurso para que sejamtrabalhadas as questões referentes à adequação do tamanho das roupas e em que o terapeuta ocupacional serve como suporte para autoconfiança do anoréxico a fazer suas escolhas e não se incomodar com os sentimentos que podem surgir durante a prova de roupas e tamanhos. ${ }^{16,19}$

0 uso de vídeos, para debate e reflexão, facilita que o terapeuta trabalhe com as questões referentes à percepção da imagem corporal que se encontra distorcida nos pacientes anoréxicos, além de ser um recurso que pode nortear a discussão dos problemas do paciente e benéfico para que os anoréxicos se informem sobre sua condição e seja reduzido o sentimento de culpa sobre a doença e possam se identificar com outros pacientes que se recuperam, mostrando-se vídeos de depoimentos de pacientes que enfrentaram a AN. ${ }^{16}$

Oficinas de autocuidado como atividades de beleza e maquiagem podem ser trabalhadas juntamente com outras atividades de imagem corporal. Geralmente essas sessões de beleza e maquiagem são utilizadas com frequência em grupo de adolescentes para abordar aspectos de gênero, individualidade e desafios da adolescência. Pois o objetivo dessa atividade é a construção de autoconfiança na área da aparência pessoal e proporcionar que a paciente estabeleça uma autoimagem adequada a sua idade de forma que proporcione qualidade de vida e saúde. ${ }^{18}$

Os pacientes anoréxicos em grande parte dos casos apresentam não satisfação de suas necessidades nas relações sociais. Nos grupos terapêuticos, um dos objetivos do terapeuta 
ocupacional é a melhoria nas habilidades sociais dos indivíduos em que se busca saber os motivos do isolamento social, trabalham-se técnicas de autoafirmação, conscientização, esclarecimento de valores e reinserção social. Um dos problemas apontados pelos anoréxicos é que se sentem desmotivados para sair de casa e ir se encontrar com amigos em um restaurante ou festa, por exemplo, pois podem estar presentes pessoas nesses ambientes que irão insistir para que eles se alimentem ou a situação de que a todo o momento serão oferecidos alimentos e relatam se sentirem incomodados com essa situação, além de se sentirem observados pelas pessoas ao saírem de casa. ${ }^{19}$

O relaxamento é citado como um recurso a ser utilizado, pois reduz os níveis de ansiedade, aumento da competência para lidar com o estresse, evitar comportamentos indesejados que aparecem devido o estresse, 0 aumento da autoestima como resultado de um maior controle sobre as reações do estresse e melhora das relações interpessoais. Porém em nenhum dos estudos foi abordado o passo a passo de alguma técnica de relaxamento, só sendo citada que existem várias técnicas comoa de respiração profunda. ${ }^{19}$

O terapeuta ocupacional juntamente com um educador físico podem realizar sessões de atividades físicas com a finalidade de ensinar níveis moderados de exercícios físicos, proporcionando fortalecimento e recondicionamento cardíaco, pois em alguns casos, os indivíduos anoréxicos realizam grande quantidade de atividade física além do que conseguem suportar. O terapeuta ajudará o paciente a reconhecer mudanças no seu corpo e sensações que mostram que a quantidade de exercício realizado é suficiente e também a organizar uma rotina diária adequada de atividade física quando já apresentam condições físicas e peso corporal adequado para prática de exercícios, se esta for uma demanda do pacientes. ${ }^{19}$

É destacado que muitas vezes, é no processo de tratamento terapêutico ocupacional que as pessoas com anorexia nervosa conseguem construir uma nova relação com seu fazer, por meio de planejar, organizar e realizar junto com 0 terapeuta suas ações cotidianas. ${ }^{23}$ Então o terapeuta ocupacional deve atuar além das intervenções citadas no resgate das capacidades e habilidades perdidas, e sim motivar o paciente a ter uma postura participativa, criativa e independente; possibilitar espaços em que seus sentimentos possam ser compartilhados e vivenciados; e organizar o desempenho de atividades de vida diária e atividades de vida cotidiana, assim como reestabelecimento dos papéis sociais e profissionais. ${ }^{23,26}$

Os melhores resultados no tratamento da AN são descritos como as que utilizaram a atividade orientada, pois está é mais eficaz do que a intervenção verbal; aqueles em que os pacientes aceitam participar do tratamento; e nos casos em que a internação, quando necessária, é precoce e composta de estratégias integradas e com a presença de uma equipe multidisciplinar. ${ }^{18,19,23}$

Apenas três estudos apontaram explicitamente o papel da Terapia Ocupacional na assistência ao tratamento da AN. Tais papéis consistem em ajudar o paciente a atingir o nível mais alto de competência psicológica, física e social; restauração de um peso corporal saudável; hábitos alimentares adequados; adaptação psicológica envolvendo a redução da fobia de peso; permitir que o anoréxico atinja o nível máximo de funcionamento psicossocial possivel; proporcionar atividades que estimulem a autoexpressão; permitir que o indivíduo com anorexia nervosa expresse suas ansiedades, medos e depressão; estimular o progresso social, intelectual e emocional; encorajar o paciente a aceitar sua nova forma e tamanho corporal; incentivar a continuidade do desempenho de funções sociais como, por exemplo, estudar; e fornecer uma avaliação profissional adequada. ${ }^{8,19,23}$

Rev. Saúde (Santa Maria), Santa Maria, v.39, n.2 p.25-34, Jul./Dez.2013 Kubota, A. M.A; et. al. ISSN 2236-5843 
Finalmente, a Terapia Ocupacional tendo como base a ciência da ocupação humana permite a seus profissionais abordagens que contemplem 0 indivíduo em atividade, pois 0 processo terapêutico passa pela organização do indivíduo em ação, indissociável de sua trajetória de vida. Considera-se como o processo de tratamento em Terapia Ocupacional a criação e conexão com as várias possibilidades de experimentar-se. Este processo deve ser guiado e orientado de acordo com a escolha do modelo ou estrutura de referência do terapeuta ocupacional. Neste trabalho, encontramos algumas delas possiveis de aplicar em pacientes com anorexia nervosa, mas fica claro a necessidade de ampliação na produção de conhecimento científico sobre o tema.

Apresentamos aqui, por meio desta revisão, alguns aspectos básicos e importantes da clínica do terapeuta ocupacional na área da Anorexia Nervosa. No entanto, salientamos que se deve reconhecer que nenhum tratamento ou abordagem isolada é efetivo para todos pacientes, e que o ganho de peso ou outros problemas estritamente relacionados com 0 diagnostico de anorexia nervosa não serão os únicos objetivos do tratamento. Por exemplo, normalmente a clínica apresenta uma limitação relacionada ao que tange a espiritualidade de seus pacientes. Lembramos que se deve considerar essa dimensão, assim como, suas experienciais sociais e culturais e os componentes observáveis do desempenho ocupacional para garantir o sucesso do tratamento e satisfação daquele que busca cuidado.

\section{Considerações Finais}

Ao realizar uma pesquisa bibliográfica sobre a AN são encontradas publicações de diferentes áreas de atuação como a Medicina, Nutrição, Psicologia, Enfermagem e Terapia Ocupacional. Porém, os estudos referentes à atuação da Terapia Ocupacional ainda não foram amplamente descritos e consolidados. Existem poucas produções científicas brasileiras específicas acerca do tema, existindo mais publicações estrangeiras. E quando se analisa as publicações no idioma português em que é citada a equipe multidisciplinar, o terapeuta ocupacional é mencionado como membro dessa equipe, porém não é descrito minuciosamente suas intervenções.

Não foi encontrado nenhum estudo sobre eficácia da intervenção do terapeuta ocupacional no tratamento de indivíduos com AN, o que indica uma carência na produção de conhecimento, com delineamento metodológico apropriado para essa finalidade. Também não sendo encontradas publicações recentes sobre o tema, nem mesmo na literatura internacional. $O$ que sugere a necessidade de que os terapeutas que trabalham com esse público publiquem estudos sobre sua prática clínica para assim permitir a busca de evidências científicas para consolidação da atuação do terapeuta ocupacional no tratamento de pessoas sofrendo de anorexia.

\section{Referências Bibliográficas}

Saúde (Santa Maria), v.39, n.2, p.23-34, 2013. Terapia ocupacional na abordagem de pessoas em tratamento por anorexia nervos

1.Pinzon V, Gonzaga AP, Cobelo A, Labaddia E, Belluzo P, Fleitlich-Bilyk B. Peculiaridades do tratamento da anorexia e da bulimia nervosa na adolescência: a experiência do PROTAD. Rev. psiquiatr. Clín. São Paulo. 2004;31(4):167-69. 
2. Doyle J, Bryant-Waugh R. Epidemiology. In: Lask B, Bryant-Waugh R. eds. Anorexia nervosa and related eating disorders in childhood and adolescence. $2^{\mathrm{a}}$ ed. East Sussex: Psychology Press; 2000. p. 41-61.

3.Cooper Z. The development and maintenance of eating disorders. In: Brownell KD, Fairburn CG. Eating disorders and obesity: a comprehensive handbook. New York: The Guilford Press; 1995. p. 199-206.

4.Salzano FT, CordásTA. Transtornos alimentares. In: CórdasTA, Salzano FT. Saúde mental da mulher. São Paulo: Atheneu; 2004. p. 201-28.

5.Habermas T. Friderada: a Case of Miraculous Fasting. Int J Eat Disord.1986;5(3):555-62.

6. Freud, S. Rascunho G: Melancolia. In: Edição Standard Brasileira das Obras Psicológicas Completas de Sigmund Freud. Rio de Janeiro: Imago, v. 1, 1985.

7.Apa. Associação Americanade Psiquiatria. Manual diagnóstico e estatístico de transtornos mentais - DSMIV-TR. $4^{\text {a }}$ ed. Porto Alegre: Artmed; 2002.

8.Oms. Organização Mundialda Saúde. Classificação Estatística Internacional de Doenças e Problemas relacionados à Saúde - CID-10. Décima revisão, v. 1. Brasília(DF), 2008 [acesso em 2013 Jan 3]. Disponível em: http://www.datasus.gov.br/cid10/N2008/cid10.htm.

9.SrinivasagamNM, Kaye W, Plotnicov KH, Greeno C, Weltzin TE, Rao R. Persistent perfectionism, symmetry, and exactness after long-term recovery from anorexia nervosa. Am J Psychiatry, United States.1995;152(11):1630-34.

10.Rastam M. Anorexia nervosa in 51 Swedish adolescents: premorbid problems and comorbidity. J Am Acad Child Adolesc Psychiatry, Suécia. 1992; 31(5): 819-29.

11. Morgan $\mathrm{CM}$, Vecchiatti IR, Negrão $\mathrm{AB}$. Etiologia dos transtornos alimentares: aspectos biológicos, psicológicos e sócio-culturais. Rev. Bras. Psiquiatr., São Paulo. 2002; 24 Suppl IIl:18-23.

12. Claudino AM, Borges MBF. Critérios diagnósticos para os transtornos alimentares: conceitos em evolução. RevBrasPsiquiatr, São Paulo. 2002; 24Suppl. III:7-12.

13. Schmidt E, Mata GF. Anorexia Nervosa: Uma Revisão. Fractal, Rev de Psico. 2008; 20(2):387-400.

14.WFTO. World FederationofOccupationalTherapy; Associação Brasileira de Terapia Ocupacional; Centro de Estudos de Terapia Ocupacional - Ceto. Definições de Terapia Ocupacional. Lins: Faculdades Salesianas de Lins, 2003.

15.MARCONI, MA; LAKATOS, EM. Metodologia do trabalho cientifico. São Paulo: Ed. Atlas, 1992. 4a ed. p. 43 e 44 .

16. Giles GM. Anorexia Nervosa and Bulimia: An Activity-Oriented Approch. The Amer. Jour. Occup. Ther., Reino Unido. 1985;39(8):510-17.

17.Souza JC, Borges PA, Ferreira RS, Gaudioso CE, Ishikawa A, Paula TH, et al. Transtornos Alimentares em Discussão Multidisciplinar. Casos Clin Psiquiatria, Campo Grande. 2002; 4(1):33-38.

18. Martin JE. Occupational Therapy in Anorexia Nervosa.J. psychrat. Res., Grã-Bretanha. 1985;19(2):45963.

19. Giles GM,Allen MA. Occupational therapy in the rehabilitation of the patient with anorexia nervosa.OccupTher in Ment Health, Inglaterra. 1986;6(1):47-66.

20.Esturaro ATA,Bucaretchi HA. Uma proposta de intervenção do terapeuta ocupacional no tratamento da anorexia nervosa. Rev de TOUSP, São Paulo. 1998; 9(2):82-7.

21. Henderson S. Frames of reference utilized in the rehabilitation of individuals with eating disorders. Can J OccupTher., Londres. 1999;66(1):43-51.

Rev. Saúde (Santa Maria), Santa Maria, v.39, n.2, p.25-34, Jul./Dez.2013

22.Matusevich D,García A, Gutt S, De La Parra I, Finkelstein, C. Hospitalization of patients with anorexia nervosa: a therapeutic proposal. EatWeightDisord., Argentina. 2002; 7(3):196-201. 
23. Morais LV. A assistência do terapeuta ocupacional para pessoas com anorexia nervosa: relato de experiência. Medicina, Ribeirão Preto. 2006.39(3):381-85.

24. Cabrera CC. Estratégias de intervenção interdisciplinar no cuidado com o paciente com transtorno alimentar: o tratamento farmacológico. Medicina, Ribeirão Preto. 2006;39(3):375-80.

25. Silva LM, Santos MA. Construindo Pontes: Relato de experiência de uma equipe multidisciplinar em transtornos alimentares. Medicina, Ribeirão Preto. 2006;39(3):415-24.

26.Quiles-Cestari LM, Ribeiro RPP. Os papéis ocupacionais de mulheres com anorexia nervosa. Rev. LatinoAm. Enfermagem, RibeirãoPreto. 2012;20(2):1-8.

27.Sepiol JM,Froelich J. Use of the role checklist with the patient with multiple personality disorder. Am J OccupTher. Massachusetts. 1990; 44(11):1008-12.

\section{Aline Midori Adati Kubota}

Endereço para correspondência - QNN 14 Área Especial, 7380. Bairro Ceilândia Sul, CEP 72220-140, Brasilia, DF, Brasil.

Email: aline.adati@gmail.com

Currículo Lattes: http://lattes.cnpq.br/6341214299801736

Recebido em 29 de maio de 2013.

Aprovado em 16 de setembro de 2013. 